\section{THE TREATMENT OF VASCULAR TUMORS BY THE INJECTION OF WATER AT A HIGH TEMPERATURE.}

WITH SUGGESTYONS AS TO ITS EMPLOYMENT IN TUBERCULAR ADENITIS, ABSCESS, FISTULOUS TRACTS, ETC.*

JOHN A. WYETH, M.D. NEW YORK.

Angiomata or non-malignant vascular tumors, especially those of large size, as cirsoid aneurisms or cavernous nevi, are among the most difficult lesions with which the surgeon has to contend. In fact, a considerable number of these cases have heretofore been deemed inoperable, and have been abandoned to an ever-increasing deformity and discomfort and, in some instances, to death from hemorrhage, pressure or other cause.

While I do not claim that the method I am about to submit is infallible or will cure every case, or is without danger, I am convinced it is worthy of serious consideration.

These vascular neoplasms may be classified on an anatomic basis as arterial, capillary and venous. The arterial angioma, or cirsoid aneurism, is most infrequently observed. The venous variety (cavernous nevus) is next in order of infrequency, while the capillary form, or "mother's mark," is most common.

In 1900 , attracted by the success which my colleague, Prof. R. H. M. Dawbarn of New York, had achieved in his operation for the starvation of inoperable tumors of the nasopharynx by dissection of all those branches of both external carotid arteries which are distributed to the regions involved, I began experiments to determine if this might not be accomplished by a simpler method, which would bring the operation within the scope of many practitioners who might hesitate to undertake such an extensive dissection in one of the so-called dangerous surgical regions. The idea in mind was to obliterate the terminal branches of the external carotid arteries by injecting into the lumen of the parent trunk below the points of origin of these branches, paraffin, alcohol, or some other substance which would cause occlusion of the vessels and render the denutrition of the tumor less difficult and more thorough.

The experiments were made on the arteries of dogs, and the substances employed were paraffin, alcohol and boiling water. Neither of the first two proved satisfactory, while the injections of water at the boiling point into the iliac of a dog caused immediate occlusion of the vessel and all of its branches. This result convinced me that with this agent the coagulation of the blood and albuminoids in vascular neoplasms was possible. The first operation was made in the following case, which had been abandoned as incurable:

Miss V. D., aged 20, consulted me in 1897 for a large cavernous nevus occupying the right lower jaw and chin and a portion of the neck immediately underneath the chin and jaw of the right side. (Fig. 1.) The tumor was congenital, had increased in size progressively with the development of the patient during her growing period, and had continued to enlarge after she had reached her full growth. Pressure of the mass had worn away the outer surface of the lower jaw, and an extensive network of enlarged veins could be seen beneath the mucous membrane of the buccal wall. The skin covering this mass of blood vessels was normal in color. She informed me that the late Dr. Hunter McGuire, Richmond, Va., had essayed to remove the tumor by operation, but had

* Read at the Fifty-fourth Annual Session of the American Medical Association, in the Section on Surgery and Anatomy, and approved for publication by the Executive Committee : Drs. A. J. abandoned it on account of hemorrhage. I repeated this effort, proceeding very cautiously, but was convinced from the extensive bleeding which followed a very small incision that the patient would perish if $\Upsilon$ persisted in the effort. The wound was packed and firm compression applied, and as soon as her condition permitted she was allowed to return home.

On July 14, 1901, under ether narcosis, an area covering one-third of the tumor was injected with from two to three ounces of boiling water. To preclude as far as possible the danger of embolism, peripheral compression was applied. With the distension of the tumor the integument became very warm to the touch, and at one time there was sufficient heat to cause my assistant, who had two fingers inserted into the mouth, to remove his hand. The medium aspirating needle was carried through the mass to within one-half of an inch of the limit of the diseased area and the boiling water forced out of the syringe until the integument turned pale under the distension. Notwithstanding the great heat there was no necrosis of the mucous membrane or the overlying integument.

The operation lasted ten minutes, and there was no pain or elevation of body temperature at any time after the patient became conscious. The blood and albuminoids were evidently coagulated. Within two weeks the area injected had diminished at least one-half in size, when a second operation was performed. At this time the injections were made through the entire remaining portions of the mass with a result equally as gratifying as at the first procedure. At the end of the second week, when the patient left me, the tumor had diminished two-thirds its former size. A third

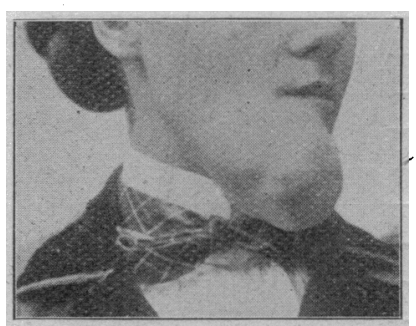

Fig. 1. - The angioma before treatment.

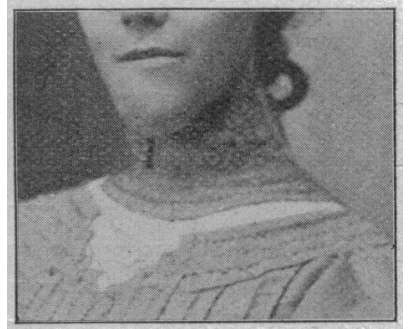

Fig. 2.-After the first and second injections. injection of a small area which had escaped coagulation was one year later performed by Dr. Stuart MeGuire, Richmond, Va., with success. The accompanying photographs show the gratifying results in this case. (Figs. 2 and 3. )

CAse 2.-A second and more difficult case of this form of angioma is that of C. G., a well-developed child of 9 years, of Lowell, Mass. At birth there was a small "mother's mark" or blue spot, about as large as a quarter of a dollar, on the back just above the right shoulder blade, a little to the right of the median line. At the age of 2 years it began to grow perceptibly, and continued to enlarge in all directions, drifting chiefly downward and to the right. In her fifth year, when the tumor was about seven inches in length and half as wide, an effort was made to remove it by operation, but on account of hemorrhage it was abandoned. The wound was packed, became infected, and resulted in the cicatrices which are shown in the photograph. The neoplasm reappeared in all parts of the operative wound, and one and one-half years later a second attempt was made, and this was also abandoned.

When she came under my observation, on Feb. 27, 1902, there was a large venous angioma occupying the back from the edge of the trapezius muscle above the right shoulder blade, over the shoulder, reaching to the middle line between the two scapulæ, then down along the posterior border of the right scapula, extending as far as the twelfth rib and well forward to the right side. The whole mass was slightly crescentic in shape, its surface was from one to two and a half inches above the level of the body, and from the elevation of the right shoulder it was evident that the abnormally enlarged vessels had burrowed between the ribs and the scapula. To the touch it was soft and compressible, the venous chan- 
nels remaining empty under pressure and refilling as soon as this was removed.

Under ether narcosis the boiling water injections were made as in the former operation, the needle being inserted in eleven different points through the neoplasm. Between five and six ounces of boiling water were thrown in. The child complained of no pain after consciousness was restored. Coagulation was evident, and within two days the area injected began to undergo diminution by granular metamorphosis. By October the entire growth had disappeared with the exception of the rounded mass, about three inches in diameter, and elevated about one and $a$ half inches above the skin, shown in the center of the area in black in the accompanying illustration. (Fig. 4.) This was injected in October, 1902, which completed the cure.

In the first operation in this case a necrosis of the skin over an area of about one inch in diameter resulted from hyperdistension with too much boiling water at this particular point. After the child left my care and returned to her home in Massachusetts this became infected and some suppuration ensued, but it in no way interfered with her recovery and the cure of this large and otherwise incurable growth.

The method has been successfully employed in arterial angioma, or cirsoid aneurism, as shown by the following case :

CASE 3.-Miss S. C., aged 27, came under my observation January, 1902. In 1885 the patient noticed a small pulsating tumor in a branch of the temporal artery situated directly over the ear. Compression on the artery in front of the ear

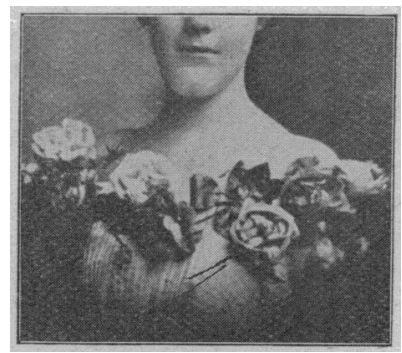

Fig. 3.-After the third injection.

by means of a cork held in place by bandage tied around the head was tried for a prolonged period. While it controlled all pulsation as long as the pressure was applied, it did not obliterate the artery or cure the angioma.

In 1886 Dr. J. M. Taylor tied the temporal artery in front of the ear, but without benefit, for after this operation the tumor increased in size. In 1887 Prof. T. G. Richardson, New Orleans, attempted to strangulate the mass subcutaneously, but without success. At a later period he introduced several large pins through the base of the tumor and surrounded it with a strong ligature. By repeated tightening of the ligature he succeeding in cutting off by strangulation a considerable section, to the base of which caustic potash was applied. The tumor, however, continued to grow, and although this attempt was repeated a year or two later by Dr. Richardson, it failed again, and any further operation was abandoned.

In January, 1902, there was a large pulsating mass covering about one-half of the left side of the scalp, which meas. ured approximately five by six inches and was elevated above the level of the normal scalp from one-half an inch to an inch. On the surface there were several large cicatrices which had resulted from the efforts of strangulation, and along the border of these scars were three or four small patches of papillomata. Leading into this tumor were five arteries, which could be seen distinctly pulsating with increasing motion as they approached their entrance to the mass. Two of these came from the left temporal, one from the right temporal and one from each occipital. The center of the angioma corresponded to the point where pulsation was first noticed. It had grown so rapidly within the year that the patient was compelled to seek relief by operation, which was done on Jan. 4,1902 , under ether narcosis, the scalp having previously been shaved and thoroughly disinfected.

The needle was entered along the course of the arteries leading into the tumor, beginning about two inches from the mass, a quantity of boiling water sufficient to coagulate these vessels being employed. It was then introduced through the tumor from side to side, injecting about a dram, withdrawing the needle for one-half of an inch and then repeating this procedure until the entire mass had ceased to pulsate. The quantity of boiling water so used was between five and six ounces. Temperature on the surface was noticed by touch, and when the heat became very perceptible to the hand and the skin began to bleach the injections in that particular area were discontinued. The warty growths noticed on the surface of the scalp were touched with the Paquelin cautery. No reaction followed the operation. The patient complained of no pain, but there was a very considerable edema of the scalp, and this, beginning on the left (the side of operation), closed the left eye and spread over the face, closing the right eye within forty-eight hours. The swelling spread as far down as the neck and was so great that had I cause to repeat a similar operation $I$ would use only about one-half

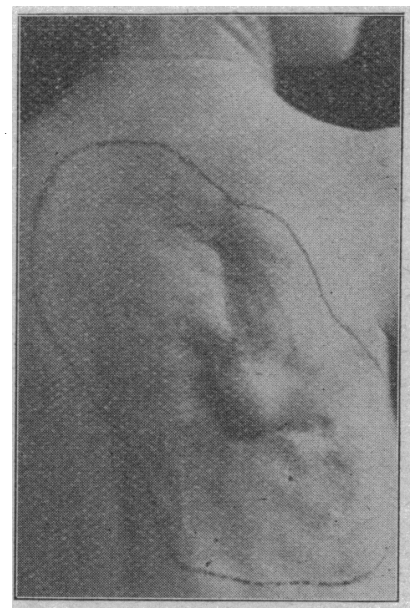

Fig. 4.-After the second injection.

the quantity of water, and would then repeat the operation after the interval of about a week.

Dr. G. C. Chandler of Shreveport, La., a brother of the patient, writes me under date of Nov. 12, 1902, that "no pulsation is present, and there is slight depression where the tumor was, owing to attrition of the skull by the long pressure of the pulsating mass. The scalp is no thicker than that covering the normal skull, and while a slight eczema persists where the tumor was situated, the hair seems to be more plentiful than before the operation."

I have employed this method successfully and without accident in a number of cases of capillary angioma, but, on account of their superficial character, they being within the substance of the integument, some cicatrization is apt to result unless very great care is taken. The weak tissues of the new growth do not offer the resistance of the normal skin which overlies the venous or arterial angiomata, and may break down under the hot water. In all of my cases the scarring has been very slight, and I think the method is well worthy of thorough trial in these cases. As they occur chiefly in children and are situated on the face, it is important to have the patient very firmly held while the injections are being made without narcosis. The legs, arms, body and head should be kept immovable, while the face should be covered by a mat in which an aperture is cut sufficient to expose the area to be injected. I take the additional precaution to have an assistant hold a sponge saturated with cold water immediately over the needle 
